\title{
RESONANT ABSORPTION OF TRANSVERSE OSCILLATIONS AND ASSOCIATED HEATING IN A SOLAR PROMINENCE. I. OBSERVATIONAL ASPECTS
}

\author{
Takenori J. Okamoto ${ }^{1,8}$, Patrick Antolin ${ }^{2}$, Bart De Pontieu ${ }^{3,4}$, Han Uitenbroek $^{5}$, \\ TOM VAN DoORSSElaere ${ }^{6}$, AND TAKAAKI YOKOYAMA ${ }^{7}$ \\ ${ }^{1}$ ISAS/JAXA, Sagamihara, Kanagawa 252-5210, Japan; okamoto@ solar.isas.jaxa.jp \\ ${ }^{2}$ National Astronomical Observatory of Japan, Osawa, Mitaka, Tokyo 181-8588, Japan \\ ${ }^{3}$ Lockheed Martin Solar and Astrophysics Laboratory, B/252, 3251 Hanover Street, Palo Alto, CA 94304, USA \\ ${ }^{4}$ Institute of Theoretical Astrophysics, University of Oslo, P.O. Box 1029 Blindern, NO-0315 Oslo, Norway \\ ${ }^{5}$ National Solar Observatory, P.O. Box 62, Sunspot, NM 88349, USA \\ ${ }^{6}$ Centre for Mathematical Plasma Astrophysics, Mathematics Department, KU Leuven, \\ Celestijnenlaan 200B bus 2400, B-3001 Leuven, Belgium \\ ${ }^{7}$ The University of Tokyo, Hongo, Bunkyo, Tokyo 113-0033, Japan \\ ${ }^{8}$ STEL, Nagoya University, Aichi 464-8601, Japan \\ Received 2015 April 12; accepted 2015 June 26; published 2015 August 11
}

\begin{abstract}
Transverse magnetohydrodynamic waves have been shown to be ubiquitous in the solar atmosphere and can, in principle, carry sufficient energy to generate and maintain the Sun's million-degree outer atmosphere or corona. However, direct evidence of the dissipation process of these waves and subsequent heating has not yet been directly observed. Here we report on high spatial, temporal, and spectral resolution observations of a solar prominence that show a compelling signature of so-called resonant absorption, a long hypothesized mechanism to efficiently convert and dissipate transverse wave energy into heat. Aside from coherence in the transverse direction, our observations show telltale phase differences around $180^{\circ}$ between transverse motions in the plane-of-sky and line-of-sight velocities of the oscillating fine structures or threads, and also suggest significant heating from chromospheric to higher temperatures. Comparison with advanced numerical simulations support a scenario in which transverse oscillations trigger a Kelvin-Helmholtz instability (KHI) at the boundaries of oscillating threads via resonant absorption. This instability leads to numerous thin current sheets in which wave energy is dissipated and plasma is heated. Our results provide direct evidence for wave-related heating in action, one of the candidate coronal heating mechanisms.
\end{abstract}

Key words: Sun: chromosphere - Sun: transition region - waves

Supporting material: animations

\section{INTRODUCTION}

For the past 15 years, transverse oscillations have been detected quite regularly in the solar corona. The TRACE observations (Handy et al. 1999), starting from 1998 showed plenty of flareinduced oscillations (Aschwanden et al. 1999; Nakariakov et al. 1999; Schrijver et al. 1999). They were successfully used for seismology of the solar corona, by estimating the magnetic field (Nakariakov \& Ofman 2001), the loop radial structure (Aschwanden et al. 2003), the vertical density scale height (Andries et al. 2005), and Alfvén transit times (Arregui et al. 2007).

Since 2007, with the advent of the CoMP instrument and the Hinode satellite (Kosugi et al. 2007), we are now convinced that the entire solar atmosphere is filled with low-amplitude propagating and standing Alfvénic waves (De Pontieu et al. 2007; Okamoto et al. 2007; Tomczyk et al. 2007), even in the absence of a flare excitation. Subsequent studies with CoMP (Tomczyk \& McIntosh 2009), Hinode and the Solar Dynamics Observatory (SDO; Pesnell et al. 2012) spacecraft revealed the wave properties and showed their presence in prominences (Schmieder et al. 2013), spicules (He et al. 2009; Okamoto \& De Pontieu 2011), and coronal loops (McIntosh et al. 2011; Wang et al. 2012; Anfinogentov et al. 2013; Nisticò et al. 2013). These oscillations are the manifestation of magnetohydrodynamic (MHD) waves (e.g., Oliver 2009; Mackay et al. 2010; Arregui et al. 2012; Mathioudakis et al. 2013 and references therein). Such waves may play an important role in the so-called coronal heating problem by supplying the Sun's hot outer atmosphere with energy (e.g., Alfvén 1947; Parnell \& De Moortel 2012). The energy carried by these Alfvénic waves is, in principle, strong enough to heat the corona and accelerate the solar wind (Uchida \& Kaburaki 1974; Antolin \& Shibata 2010; van Ballegooijen et al. 2011; Matsumoto \& Suzuki 2014). However, although such waves are sometimes damped on short spatial and temporal scales in observations (Aschwanden et al. 1999; Pascoe et al. 2010, 2011), it is still unclear whether any significant dissipation (and thus heating) occurs in the corona, which has very low resistivity.

The dissipation of transverse MHD waves in an inhomogeneous medium has been studied for decades (e.g., Ionson 1978; Hollweg et al. 1990; Sakurai et al. 1991; Goossens et al. 1992; van Doorsselaere et al. 2004). Theoretically, it is expected that the transverse, coherent motion is subject to damping by resonant absorption in an inhomogeneous prominence thread (Arregui et al. 2008; Soler et al. 2012). Resonant absorption converts the transverse wave into azimuthal motions with a fine spatial scale in the tube's boundary layer (Soler et al. 2010; Verth et al. 2010; Arregui et al. 2011). The resonant and amplified azimuthal motions are prone to be unstable to the Kelvin-Helmholtz instability (KHI) because of the large shear motions. The KHI deforms the boundary layer and leads to enhanced dissipation of the wave energy into heat in thin, turbulent current sheets (Karpen et al. 1993; Ofman et al. 1994; Lapenta \& Knoll 2003; Terradas et al. 2008a). Recent 
(a) $\mathrm{Ca} I \mathrm{H}$
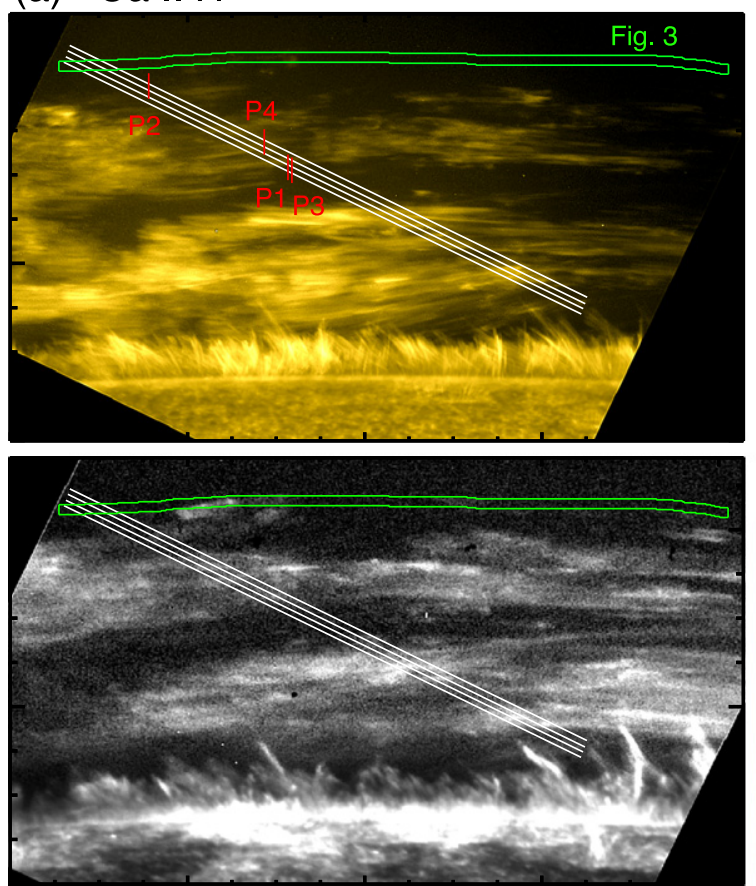

(b) Si IV (c) $\mathrm{Mg} \mathrm{II} \mathrm{k}$
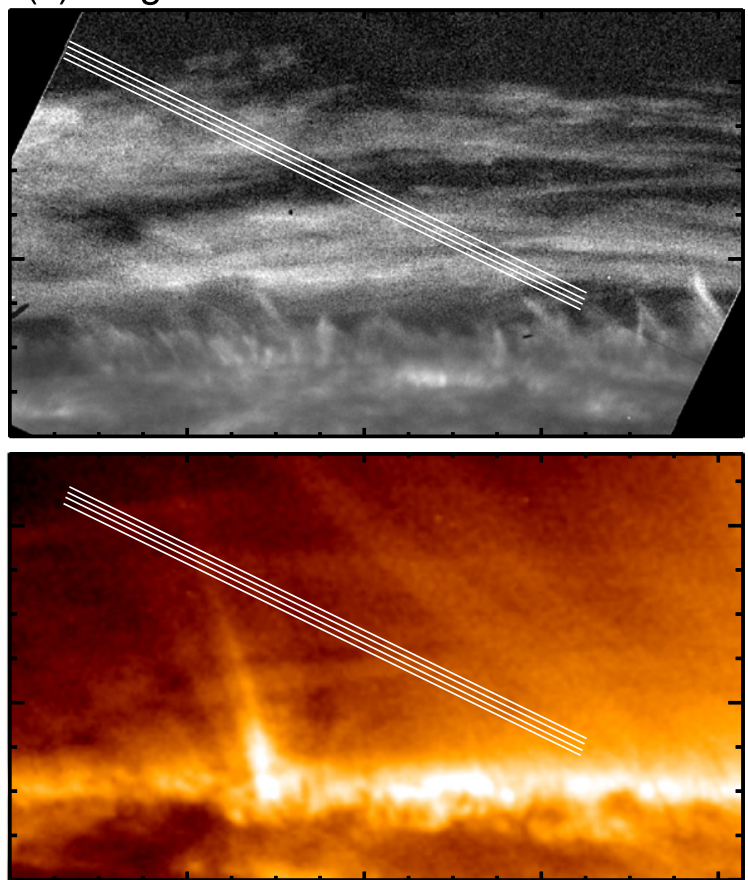

(d) Fe IX
$10,000 \mathrm{~km}$

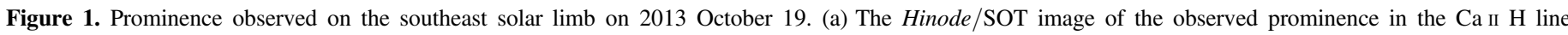

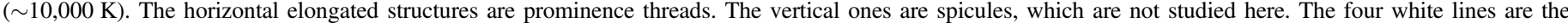

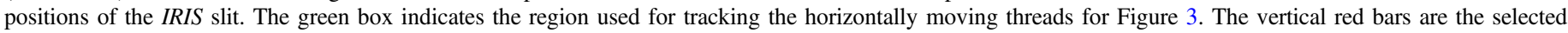

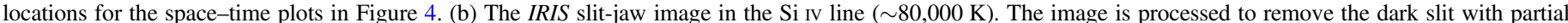

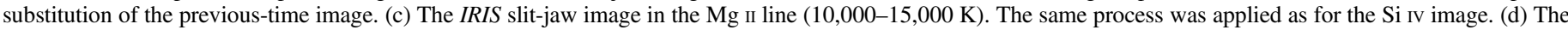

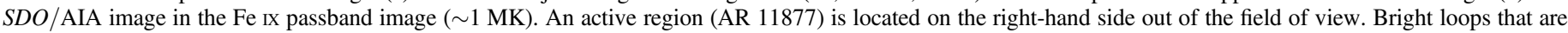
superposed in front of the prominence along the LOS come from the edge of the active region.

(An animation of this figure is available.)

simulations by Antolin et al. (2014) show that even small amplitude oscillations can lead to the instability, whose vortices combined with line-of-sight (LOS) effects result in strand-like structures within coronal loops, but at scales that are difficult to resolve with current coronal instruments.

This study is composed of two parts. In this first paper, unique coordinated observations with the Solar Optical Telescope (SOT; Shimizu et al. 2008; Suematsu et al. 2008; Tsuneta et al. 2008) of Hinode and NASA's recently launched Interface Region Imaging Spectrograph (IRIS; De Pontieu et al. 2014) satellite are used to provide evidence for a telltale sign of resonant absorption and associated heating in an active region solar prominence. We extend the three-dimensional MHD model used in Antolin et al. (2014) to a scenario matching our observations. Through radiative transfer, we show that the observed transverse dynamical coherence, the specific phase relations between the azimuthal motions and displacement of the threads, and the characteristic heating are characteristic features of resonant absorption combined with the KHI. The details of the numerical model and a more thorough analysis of the observational signatures of transverse MHD waves in prominences are provided in Antolin et al. (2015), hereafter Paper II.

\section{OBSERVATION AND DATA REDUCTION}

IRIS observed an active region prominence on the southeast limb of the Sun from 09:00 UT to 11:00 UT on 2013 October
19 (Figure 1). Four-step sparse raster scans, which have a $1^{\prime \prime}$ gap between neighboring slit positions, were performed together with the slit-jaw images of the $\mathrm{Si}$ IV and $\mathrm{Mg}$ II lines. The cadence was $10 \mathrm{~s}$ for the slit-jaw images and $20 \mathrm{~s}$ for spectra at each position with the spatial and spectral resolutions of $0^{\prime \prime} .33-0 "$. $40\left(240-290 \mathrm{~km}\right.$ on the Sun) and $3 \mathrm{~km} \mathrm{~s}^{-1}$. The exposure time was $4 \mathrm{~s}$ in both. Coordinated observations with the SOT were performed from 09:09 to 10:27 UT. The SOT obtained $\mathrm{Ca}$ II $\mathrm{H}$-line filtergrams with a cadence of $8 \mathrm{~s}$, exposures of $1.2 \mathrm{~s}$, and the spatial resolution of 0 ". $2(150 \mathrm{~km})$. The Atmospheric Imaging Assembly (AIA; Lemen et al. 2012) on board SDO provided context filtergrams of multiple wavelengths with a cadence of $12 \mathrm{~s}$.

Here we used level-2 data of IRIS, which are calibrated by dark current subtraction, flat-field correction, and geometrical correction. Remaining offsets by a few pixels between the slitjaw images and spectral data were found, and then we shifted the images to align with fiducial marks that appear on the slitjaw images and the spectra. Next, we performed a co-alignment of the time series of the slit-jaw images. Large-amplitude orbital variation of IRIS pointings, which is called wobble, is typically removed at the moment of planning (De Pontieu et al. 2014), but a small misalignment of less than $1^{\prime \prime}$ still remained. With the cross correlation of two neighboring-time images of Si IV, the offset information was derived and applied for all IRIS slit-jaw and spectral data. Hence, one can find slow drifts of the slit locations in the time series of images (see the animation of Figure 1). Although this impact was small enough 
in a timescale of focused oscillations (10-20 minutes), we considered it when we chose the nearest locations to investigate the LOS velocity of oscillating threads. Lastly, we performed co-alignments between the IRIS slit-jaw images (Si IV) and the SOT filtergrams (Ca II) by adjusting their plate scales, rotating, and shifting images. To emphasize the faint prominence structures, a radial-density filter was applied for the disk parts (also part of spicules) of all images. No gamma value is applied for these images.

We mainly use Mg II k (2796 $10,000 \mathrm{~K})$ spectra of the prominence to determine their LOS velocity as a function of time. Even though the spectra show some central reversal, we perform centroiding to determine the LOS velocity which provides a good estimate of the velocity (Leenaarts et al. 2013). Single Gaussian functions are used to fit the Mg II spectra to determine the centroid LOS velocity. Comparison of these LOS velocity signals with oscillatory displacements of the same threads in the plane of sky (POS), obtained in the $\mathrm{Ca}$ II $\mathrm{H}(3968 \AA, 10,000 \mathrm{~K})$ passband of the SOT, allows us to reconstruct the three-dimensional flow generated by the waves.

\section{METHODS AND RESULTS}

\subsection{Height Dependence of Threads and Signatures of Heating}

The $\mathrm{Ca}$ II $\mathrm{H}$ line animation of Figure 1 shows that the prominence consists of numerous horizontal threads that move horizontally with various speeds $\left(10-40 \mathrm{~km} \mathrm{~s}^{-1}\right)$. Space-time plots tracking the flow reveal that many of these threads oscillate in the POS, with signatures of damping. These features can also be seen in the Mg II k and Si IV (1403 $\AA$, $80,000 \mathrm{~K})$ IRIS images, despite the coarser spatial resolution. In the space-time plot of LOS velocity, derived from $\mathrm{Mg}$ II $\mathrm{k}$ spectra along one slit location, we can find oscillatory patterns at all heights (Figure 2(b)). Moreover, the upper region shows higher velocity amplitudes than the lower region. Similarly, the line width of $\mathrm{Mg}$ II $\mathrm{k}$ is larger in the upper region than in the lower region (Figure 2(c)). From the histogram of LOS velocity (Figure 2(d)), the higher region (regions 3 and 4, indicated by yellow and red lines, respectively) has more high-speed components with more than $15 \mathrm{~km} \mathrm{~s}^{-1}$, while velocity in the lower region is more confined around $0 \mathrm{~km} \mathrm{~s}^{-1}$. This is consistent with the large-amplitude motions seen in the Ca II animation of Figure 1. Similarly, the histogram of line width (Figure 2(e)) also shows stronger broadening in the higher region than in the lower region. We note that the line width in Figure 2 includes the instrument line broadening $\left(5.5 \mathrm{~km} \mathrm{~s}^{-1}\right.$ in FWHM). The animation shows that the lower region has more LOS superposition with a multitude of threads overlapping. One would expect such superposition to lead to broader spectral line profiles. However, this is not found, suggesting that the difference in non-thermal line broadening between the low and high regions is instead caused by increased turbulence or unresolved wave motions, which can lead to heating.

Further careful investigation reveals that the horizontal threads in $\mathrm{Ca}$ II $\mathrm{H}$ have smaller lengths and shorter lifetimes at higher altitudes. As the moving threads in the $\mathrm{Ca}$ II line fade away, co-spatial threads appear in the hotter Si IV line with similar horizontal speeds. Space-time plots of the $\mathrm{Ca}$ II and Si IV images show this more clearly (Figure 3). Even though some locations have coexistence of cooler and hotter materials, trajectories of several threads show the transition from lower to higher temperature. These finally fade out. This indicates that the cool plasma is heated to coronal temperature through the mid-temperature range of the $\mathrm{Si}$ IV line.

Two kinds of intensity variability can be observed in the animation of Figure 1. First, is a variability produced by the dynamic nature of threads linked to flows and waves. This effect, as discussed in the next section, is mostly behind the fact that a fixed slit can only capture parts of the transverse oscillations in the POS, and entails a timescale on the order of one period or less. The second kind of variability corresponds to a gradual intensity change from the chromospheric emission in $\mathrm{Ca}$ II $\mathrm{H}$ to emission in the transition region range of the $\mathrm{Si}$ IV line, and can be appreciated in Figure 3. This variability is therefore linked to heating and occurs on slightly longer timescales of one to two periods. Both effects, dynamics and heating, are intertwined and make the exact determination of lifetimes of threads difficult, even more from the strong LOS projection effects (especially in $\mathrm{Ca}$ II $\mathrm{H}$ ). In the animation of Figure 1, we observe threads appearing and disappearing in the top part of the prominence on timescales of 10-15 minutes, i.e., a couple of periods of the oscillations. Such lifetimes appear to be longer in the lower part of the prominence, where projection effects are stronger, but also where dynamics are reduced, as explained previously.

\subsection{Transverse Oscillations}

The Ca II animation of Figure 1 clearly shows the ubiquity of transverse motions of threads. Oscillations over several periods are not easy to spot in this kind of dynamic prominence. This is largely due to the horizontal flow, which makes the oscillations appear non-stationary (Okamoto et al. 2007), and move them quickly away from the fixed slit positions. Also, as shown by Figure 4 and the Ca II animation of Figure 1, vertical displacements of large horizontal sections are observed. Both effects complicate the visibility of multiple periods. To clearly indicate the presence of transverse oscillations and better visualize the longer periodic oscillations of the threads, spacetime plots at different horizontal locations following the flow in the threads are provided in the animations of Figure 4. One can clearly find two- to three-period oscillations of P1 and P3 threads with damping of their amplitudes (marked by arrows in the animations). Although the abundance of oscillations is also clear for P2 and P4, the large quantity of threads and their very dynamic nature impedes a fully clean tracking of oscillations beyond two periods. Signatures of damping are detected, but also constant and even, in some cases, increasing amplitude oscillations are detected.

\subsection{Characteristic Phase Differences and Dynamical Coherence}

Next, we focus on four oscillating cool threads that can be seen clearly and individually with little superposition in the $\mathrm{Ca}$ II images (P1-P4 in Figure 1(a)) to investigate the phase relationship between the transverse displacement in the $\mathrm{Ca}$ II line and LOS velocity in the Mg II line (Figure 4). We show the combined velocity signal from the nearest slit location at each time. Despite the fact that the threads oscillate across the IRIS slit locations at all times, the LOS velocity signals detected around the threads are strongly coherent (Figure 5). Interestingly, the oscillating threads show phase differences around $90^{\circ}$ and $180^{\circ}$ between the transverse displacement in the POS 
(b) LOS velocity $(\mathrm{km} / \mathrm{s})$

(a) $\mathrm{Ca} I \mathrm{H}$

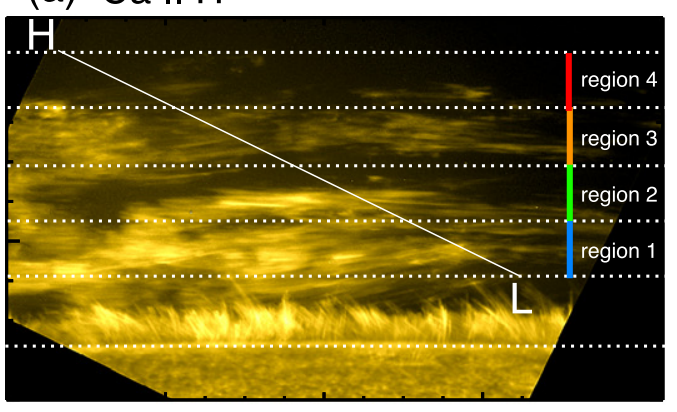

$10,000 \mathrm{~km}$
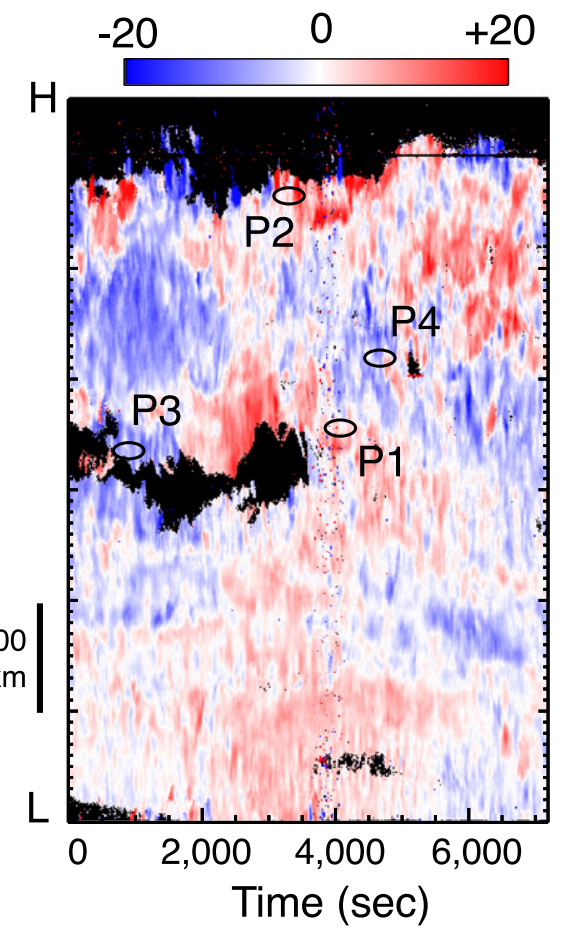

(c) Line width $(\mathrm{km} / \mathrm{s})$

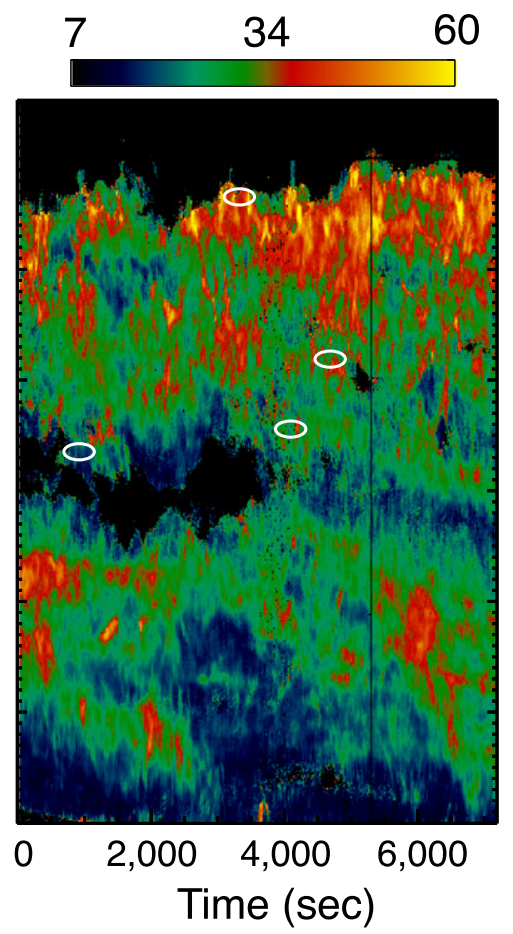

(d) Probability density of LOS velocity with height

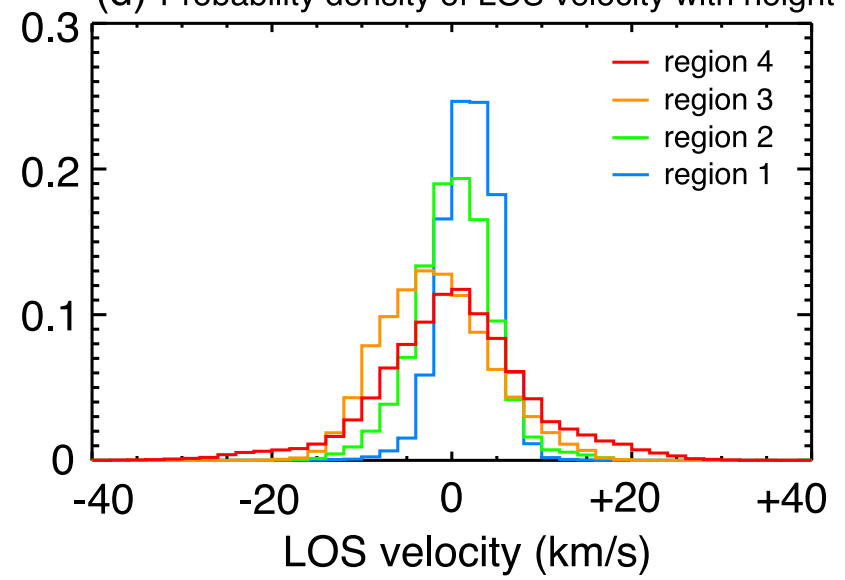

(e) Probability density of line width with height

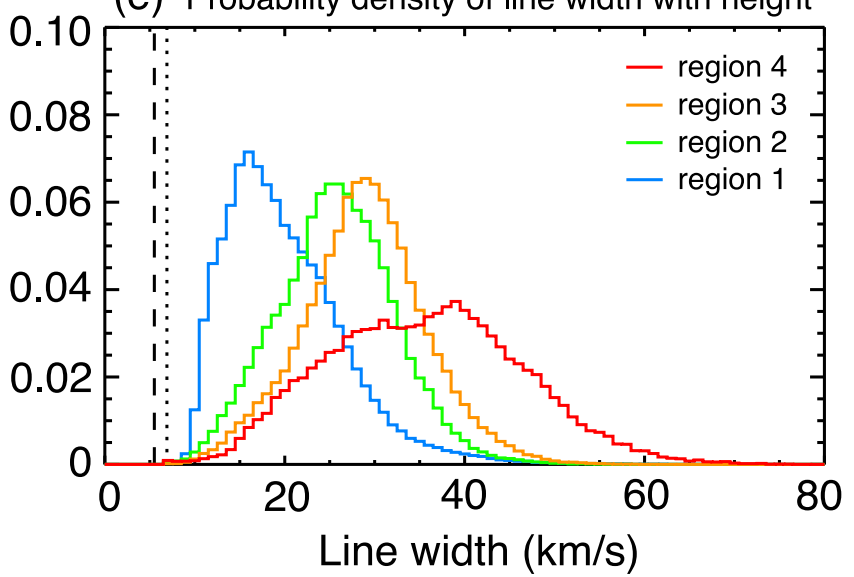

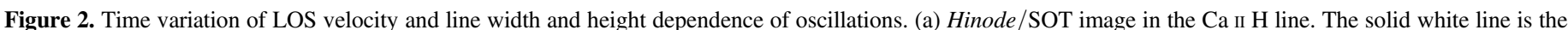

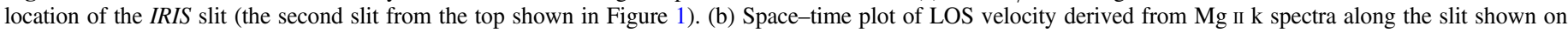

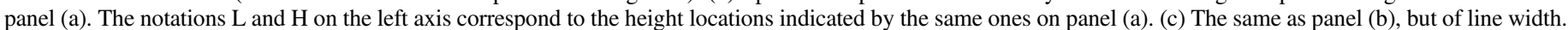

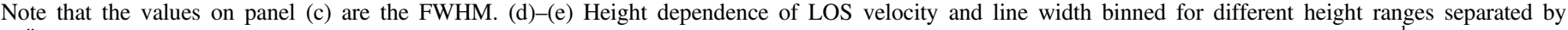

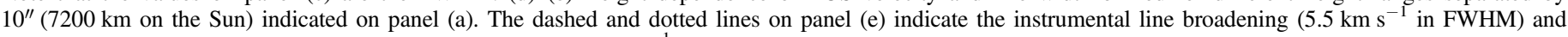
combination of the instrumental and thermal broadenings $\left(6.9 \mathrm{~km} \mathrm{~s}^{-1}\right.$ in FWHM), respectively.

and the LOS velocity. Large phase differences above $90^{\circ}$, in particular $180^{\circ}$, do not match theories of the classic kink mode with a discontinuous boundary in the density profile, nor those invoking classic axisymmetric torsional Alfvén waves or rotation (Goossens et al. 2014).

Figure 5 shows the locations of the slits around the threads for positions $\mathrm{P} 1-\mathrm{P} 4$. Besides the out-of-phase relation between the POS motion of the thread and the LOS velocity, another striking feature is the existence of coherence in the Doppler signal across a significant transverse distance, on the order of a thread diameter or more, away from (above and below) the $\mathrm{Ca}$ II $\mathrm{H}$ thread. Such coherence may come as a surprise if one assumes that the external medium dominates our signal-if that were the case, one would expect a loss of coherence. Besides coherence in the LOS velocity, the existence of coherent transverse POS motion can also be clearly seen in the animations of Figure 4. Such coherent POS motion of threadlike structure seems to be a common characteristic of transverse waves in prominences (Okamoto et al. 2007; Lin et al. 2009; Lin 2011; Hillier et al. 2013) and in loops, as evidenced by siphon flows (Ofman \& Wang 2008) or coronal rain (Antolin \& Verwichte 2011). In the present observations, we show evidence, for the first time, that the coherent motion extends to the azimuthal flow generated by the transverse wave.

It is important to note that the $\mathrm{Ca}$ II $\mathrm{H}$ threads appear to be surrounded by $\mathrm{Mg}$ II $\mathrm{k}$ emission, as evidenced by Figure 1. This 

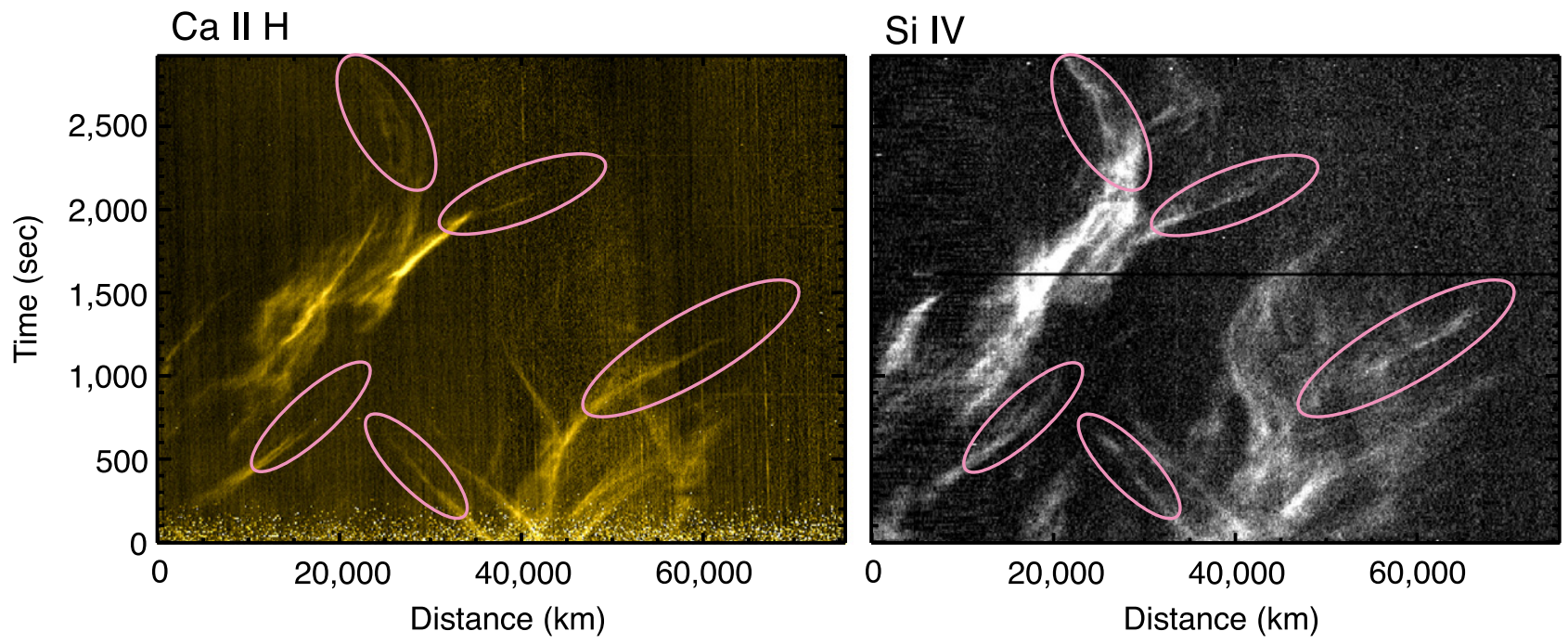

Figure 3. Heating of prominence threads. Left: space-time plot of Hinode/SOT Ca II image $(\sim 10,000 \mathrm{~K})$ along the green box shown in Figure 1. The intensity in the green box is summed vertically to include the entire part of oscillating threads. Right: the same as the left panel, but for the IRIS Si IV image ( $\sim 80,000 \mathrm{~K})$. Several paths (indicated by circles) show the transition of temperature from cool to hot, as the threads in the Ca II line fade away and co-spatial threads appear in the Si Iv line.

(a) P1

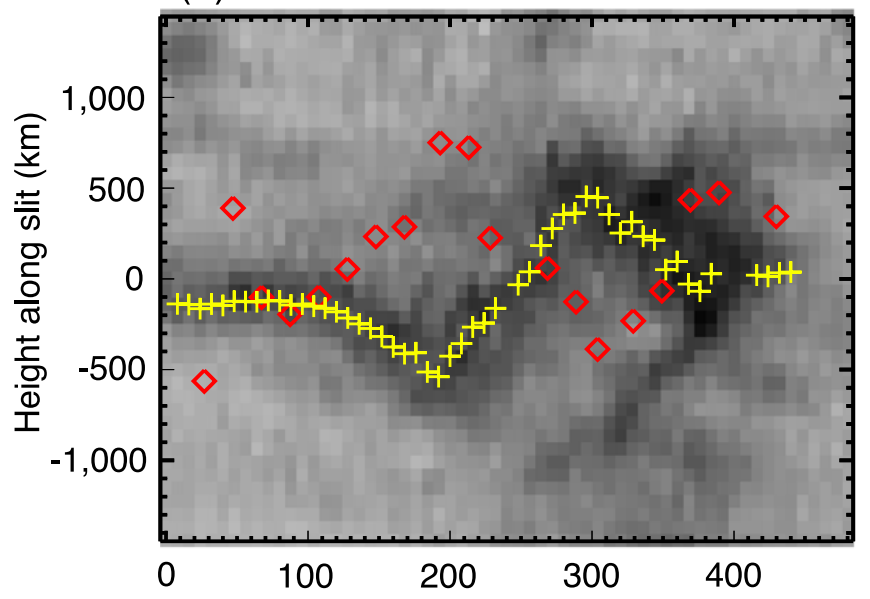

(c) P3

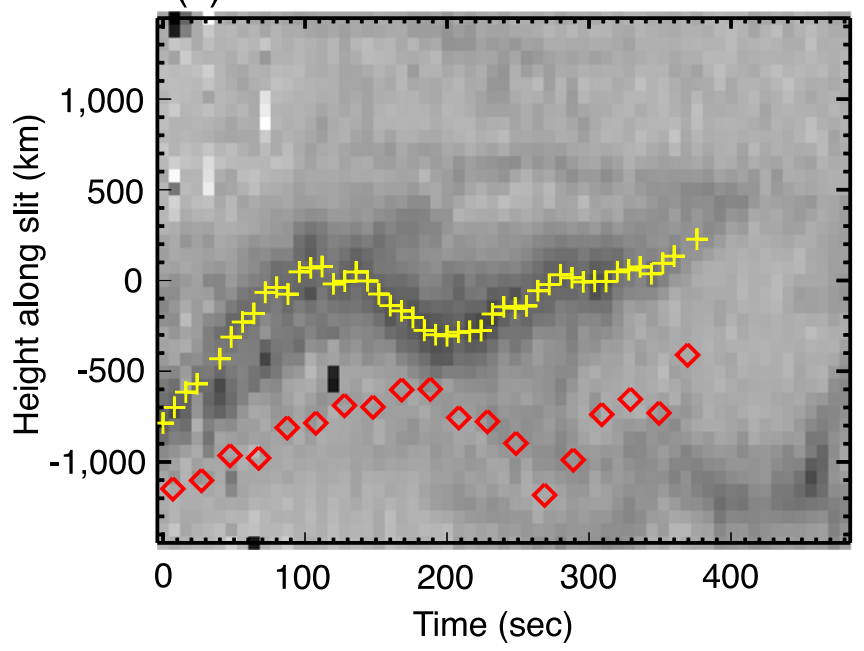

(b) P2

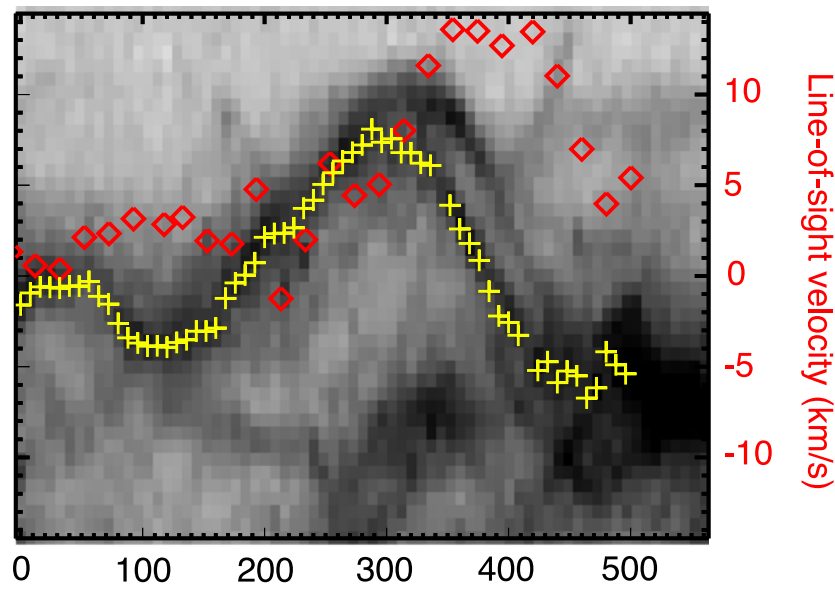

(d) $\mathrm{P} 4$

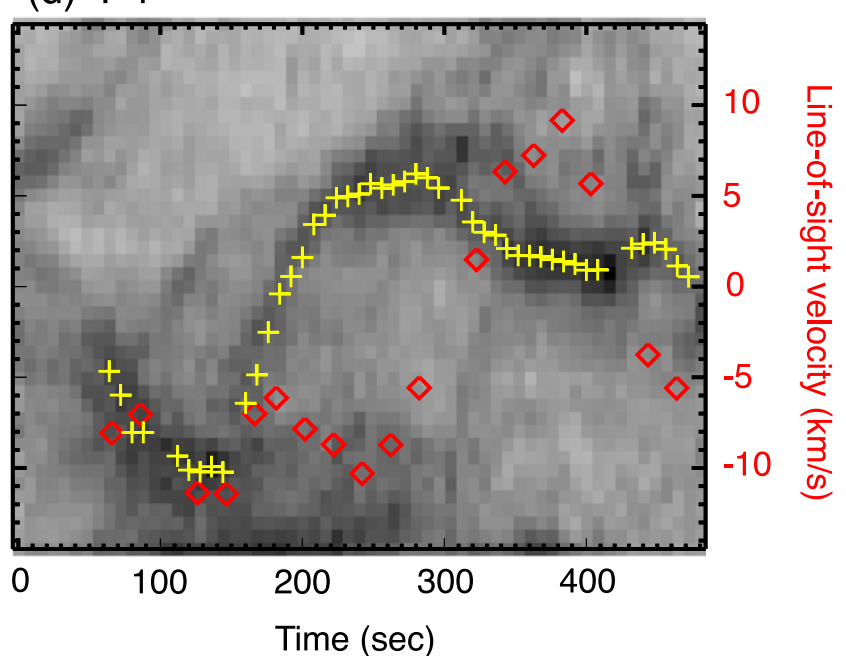

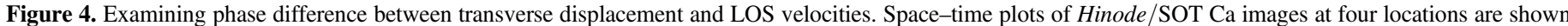

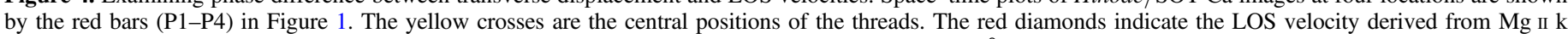

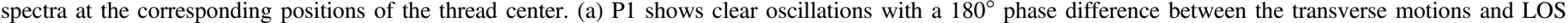
velocities. (b) P2 presents similar oscillations, but with a $90^{\circ}$ difference. (c)-(d) P3 and P4 show phase differences between $90^{\circ}$ and $180^{\circ}$.

(Animations (a, b, c, and d) of this figure are available.) 

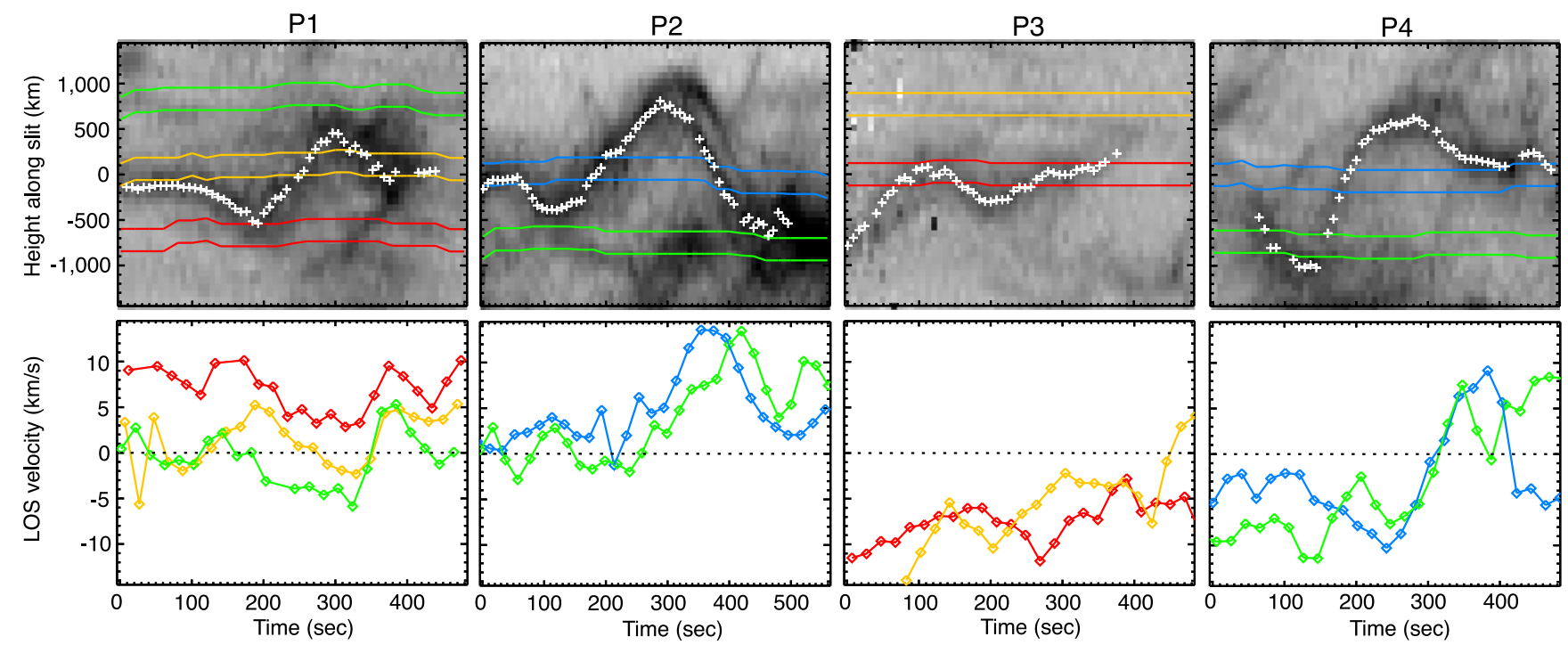

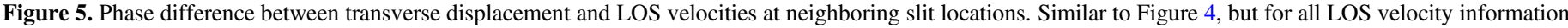

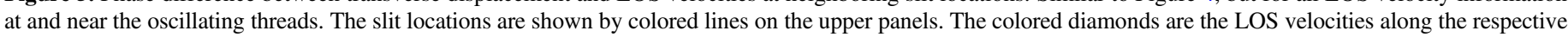
colored slits. We can find some coherence of the velocity patterns over a significant distance away from the threads.

fact, together with the existence of dynamical coherence in the POS and along the LOS at a significant distance across the threads suggests the existence of larger prominence flux tubes containing the threads. This scenario will be further explored in the next section.

\subsection{The Transverse MHD Wave Model}

To understand the observational results and, in particular, the peculiar phase difference, we use three-dimensional MHD simulations of a transversely oscillating prominence flux tube, combined with radiative transfer modeling. The flux tube has initial internal to external electron number density $\left(n_{i} / n_{e}\right)$ and temperature $\left(T_{i} / T_{e}\right)$ ratios of 10 and $1 / 100$, respectively. The transition layer between the internal and external medium has a width of $l / R \approx 0.4$, where $R$ is the flux tube radius. For the forward modeling, we give typical values found in prominences $n_{i}=10^{10} \mathrm{~cm}^{-3}$ and $T_{i}=10^{4} \mathrm{~K}$ (Tandberg-Hanssen 1995; Vial \& Engvold 2015). At time $t=0$, the flux tube is subject to a transverse perturbation, generating a fundamental standing transverse MHD wave with longitudinal wavenumber $k \approx 0.015 / R$, amplitude $v_{0}=8 \mathrm{~km} \mathrm{~s}^{-1}$, and a phase speed of $776 \mathrm{~km} \mathrm{~s}^{-1}$. The external plasma- $\beta$ is 0.01 and the fully ionized external medium has a magnetic field value of $18.6 \mathrm{G}$. We assume a long $(L=200 \mathrm{Mm})$ and thin $(R=1 \mathrm{Mm})$ prominence flux tube, matching the observations. For further details, please see Paper II.

As expected from theory, resonant absorption in the simulation sets in immediately and the energy from the purely transverse kink mode is rapidly transferred to the azimuthal waves in the resonant layer at the boundary of the tube (see the animation of Figure 6). This induced azimuthal component of the velocity is, at first, in phase with the dipole-like azimuthal flow outside of the tube, thus exhibiting a $90^{\circ}$ phase difference with the transverse displacement (Goossens et al. 2014). Since the Alfvén speed increases as the density decreases the further away we are from the tube's axis, but especially across the boundary layer, the periods of oscillation of the azimuthal waves in the resonant layer will be smaller than that of the transverse displacement, leading to a drift in time between the transverse displacement and LOS velocity (a process known as phase mixing). Over long timescales of several periods, this process leads to the generation of small scales from which a broadening of the line width, a decrease of the Doppler signal, and a loss of coherence is obtained across the resonant layer. In the short observable window of a few periods following an external perturbation (before resonant absorption dampens out the transverse POS motion) a favored $180^{\circ}$ phase difference stands out. This phenomenon is more clearly seen with a standing wave, for which the phase difference cannot grow indefinitely due to the limited length of the flux tube (Figure 6, see also the schematic representation in Figure 7(a)).

Apart from resonant absorption, another important mechanism in our simulations is the KHI. As in the coronal case (Terradas et al. 2008a; Antolin et al. 2014), the KHI is rapidly triggered at the boundaries after one period of oscillation, leading to the generation of vortices and current sheets (see Figure 2 in Paper II). Importantly, a complex interplay between resonant absorption and KHI sets in, in which the instability extracts energy from the resonant layer and imparts momentum on the generated eddies, which rapidly degenerate into turbulent-like flows, as can be seen in Figure 6's animation.

To analyze the overall effect of resonant absorption combined with KHI, we performed detailed radiative transfer modeling, explained in the next section.

\subsection{Radiative Transfer}

The chromospheric conditions of our flux tube demand a completely different approach in terms of forward modeling with respect to the previously modeled coronal case (Antolin et al. 2014). In order to properly compare with observations, we translate the numerical results into observable quantities by means of appropriate radiative transfer modeling. For optically thin transition region lines such as Si IV $1402.77 \AA$, we calculate the synthetic emission using the FoMo code (Antolin \& van Doorsselaere 2013), based on the CHIANTI atomic database version 7 (Dere et al. 1997, 2009). For optically thick lines such as $\mathrm{Mg}$ II $\mathrm{h}$ and $\mathrm{k}$ and $\mathrm{Ca}$ II $\mathrm{H}$ and $\mathrm{K}$, we use the $\mathrm{RH}$ code, as follows. We employed the two-dimensional version of 

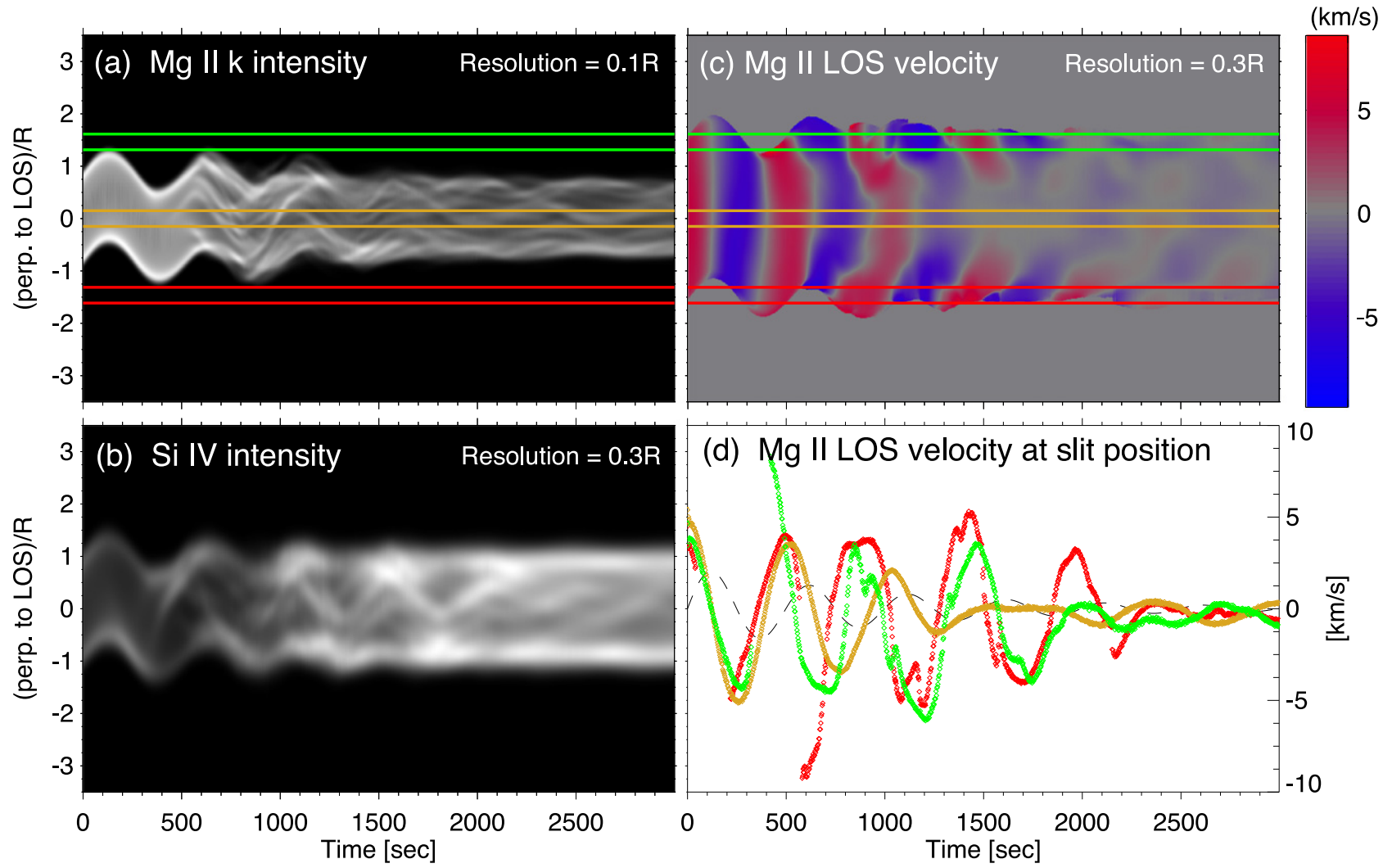

Figure 6. Synthetic intensity and LOS velocity from a simulated prominence flux tube with a transverse oscillation for an LOS angle of $45^{\circ}$ in the plane perpendicular to the tube axis. (a) Intensity of the $\mathrm{Mg}$ II $\mathrm{k}$ line with a spatial resolution of $0.1 R$ ( $R$ being the radius of the tube). (b) Intensity of the $\mathrm{Si}$ iv line with a spatial resolution of $0.3 R$. (c) Space-time plot of the LOS velocity for a slit perpendicular to the tube axis located at the center of the tube with a spatial resolution of $0.3 R$. An arrow shaped pattern develops due to resonant absorption. (d) Doppler signals along the green, orange, and red slit locations, with respective colors (whose colored edges are shown in panels (a) and (c)), centered at a distance of $1.5 R, 0 R$ and $-1.5 R$ from the tube's center, respectively. The black dashed line corresponds to the transverse displacement of the tube in the POS, calculated from Gaussian fits to the intensity image (a) for each time step. Times without Doppler signal correspond to regions for which the signal is too low. The phase differences between the LOS velocities and POS displacements match those in the observations (Figure 4).

(An animation of this figure is available.)

the RH code (Uitenbroek 2001, based on the method described in Rybicki \& Hummer 1992) to calculate the emergent spectra of the $\mathrm{Mg}$ II $\mathrm{h}$ and $\mathrm{k}$ as well as the $\mathrm{Ca}$ II $\mathrm{H}$ and $\mathrm{K}$ resonance lines in non-LTE through a slice perpendicular to the axis of the prominence. The $\mathrm{RH}$ code uses the efficient Accelerated Lambda Iteration scheme (Rybicki \& Hummer 1992) for transitions with overlapping wavelengths. It uses a formal solution of the transfer equation based on the short-characteristics method (Kunasz \& Auer 1988) with Bezier interpolation (Auer 2003).

Since the MHD code does not provide explicit electron number densities (required to calculate the collisional excitation, de-excitation, and ionization-recombination rates), they were computed with the RH code in a two-step process. First, an estimate of electron densities was made under the assumption of LTE. Second, this first estimate was used to calculate a full two-dimensional non-LTE solution of a sixlevel plus continuum hydrogen atom. Finally, the ionization of hydrogen (i.e., the proton density) gives an estimate of the nonLTE ionization contribution of hydrogen, which was added to the LTE contribution of all other elements (including $\mathrm{He}$ ).

We used abundances from Grevesse \& Anders (1991) for our calculations, which correspond to photospheric values. For the atomic models, we employed the five-level plus continuum model for Ca II (Uitenbroek 1989), the ten-level plus continuum model for Mg II (Uitenbroek 1997), and a five-level plus continuum model for Hydrogen with transition values (Vernazza et al. 1981), treating all pertinent radiative boundbound and bound-free transitions explicitly. Since the prominence cross section is optically thick, only in the very cores of the lines, we used the approximation of complete frequency redistribution in all line transitions, including the Lyman lines in hydrogen. This is justified because wavelengths outside the Doppler core are optically thin and scattering in them does not significantly affect the line transfer in the line cores.

\subsection{RH Results}

Through forward modeling of the numerical results, we generate observable quantities for direct comparison with the IRIS and Hinode observations. We first calculate the synthetic emission in $\mathrm{Mg}$ II $\mathrm{h}$ and $\mathrm{k}$ and $\mathrm{Ca}$ II $\mathrm{H}$ and $\mathrm{K}$ with $\mathrm{RH}$ on a twodimensional cross-section at the center of the tube at a given time during the numerical simulation (we pick time $t=1,162$ $\mathrm{s}$, corresponding to roughly two full periods from the start of the simulation; the numerical model at this time is shown in Figure 2 in Paper II). In Figure 8, we show the source function at the center wavelengths of the $\mathrm{Mg}$ II $\mathrm{k}$ and $\mathrm{Ca}$ II $\mathrm{H}$ lines, as well as the contours for different values of the optical thickness. As can be seen, the source function shows a ring shape with maximum values around the boundary layer of the tube and 

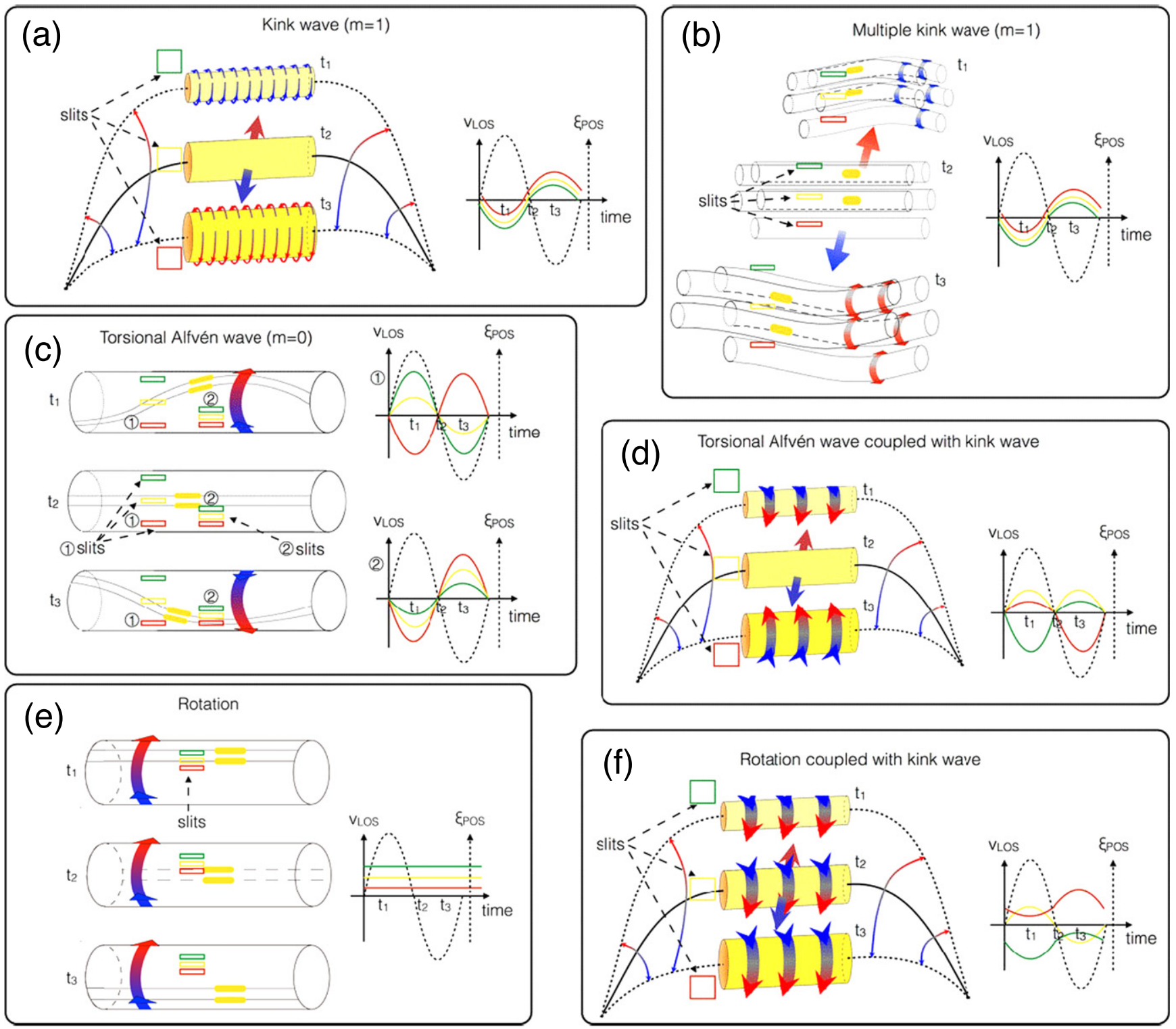

Figure 7. Schematic representation of the physical model explaining our observations and other considered interpretations. (a)-(b) The transverse MHD wave model: a single flux tube and multiple flux tubes subject to a kink wave. (c)-(d) Flux tube with a torsional Alfvén wave alone or coupled with a kink wave. (e)-(f) Flux tube with rotation alone or coupled with a kink wave. The observed threads can each correspond to a flux tube (a), (d), (f) or to high density regions within a flux tube (b), (c), (e). Locations of the IRIS slit are sketched as green, yellow, and red boxes, as in the observations (5). The width of the slit corresponds to that of the thread. The predicted Doppler signal at each slit location (solid) and the POS motion (dashed) in time is sketched on the right side in each panel. For (c), two cases can be distinguished depending on the location of the slits over the flux tube. In each case the middle position is the equilibrium position for an oscillation. In the top/bottom position, the thread is the furthest away/closest from/to the observer. For the transverse MHD wave model (a), (b) the azimuthal motions result in a blue/redshift at the topmost/bottom position (blue/red arrows), as observed. None of the other scenarios match the observations.

negligible values at the center of the tube. This means that the emergent intensity in both of these lines comes from the boundary of such flux tubes.

\section{DISCUSSION}

\subsection{A Ring Shape for the Source Function}

The obtained ring shape for the source function in $\mathrm{Mg}$ II $\mathrm{h}$ and $\mathrm{k}$ and $\mathrm{Ca}$ II $\mathrm{H}$ and $\mathrm{K}$ is mostly a temperature effect. The cool prominence plasma around the boundary layer is heated up by the KHI to transition region temperatures, thereby inwardly enlarging the transitional layer from the core to the surrounding corona. The higher temperature in the outer ring raises both the electron density and the electron temperature, both giving rise to more collisional excitations in the $\mathrm{h}$ and $\mathrm{k}$ lines (that are then followed by an increased rate of spontaneous emission). The radiative transfer results further suggest that cool chromospheric cores of such flux tubes do not contribute to the intensity in $\mathrm{Mg}$ II $\mathrm{h}$ and $\mathrm{k}$ but only to opacity. The opacity in these lines depends on the ground level populations in $\mathrm{Mg}$ II and not on the radiation field. Also, the emerging intensity is optically thin and is therefore mostly dependent on temperature. Accordingly, rays going through the center of the tube are optically thick (in $\mathrm{k}$ more than in h, by a factor of two); thus, these suffer from self absorption of the signal that comes from the back surface. Such rays have an intensity ratio of $\mathrm{k}$ and $\mathrm{h}$ lines tending to 1.5 . On the other hand, rays going through the surface of the tube only have 
Mg II k

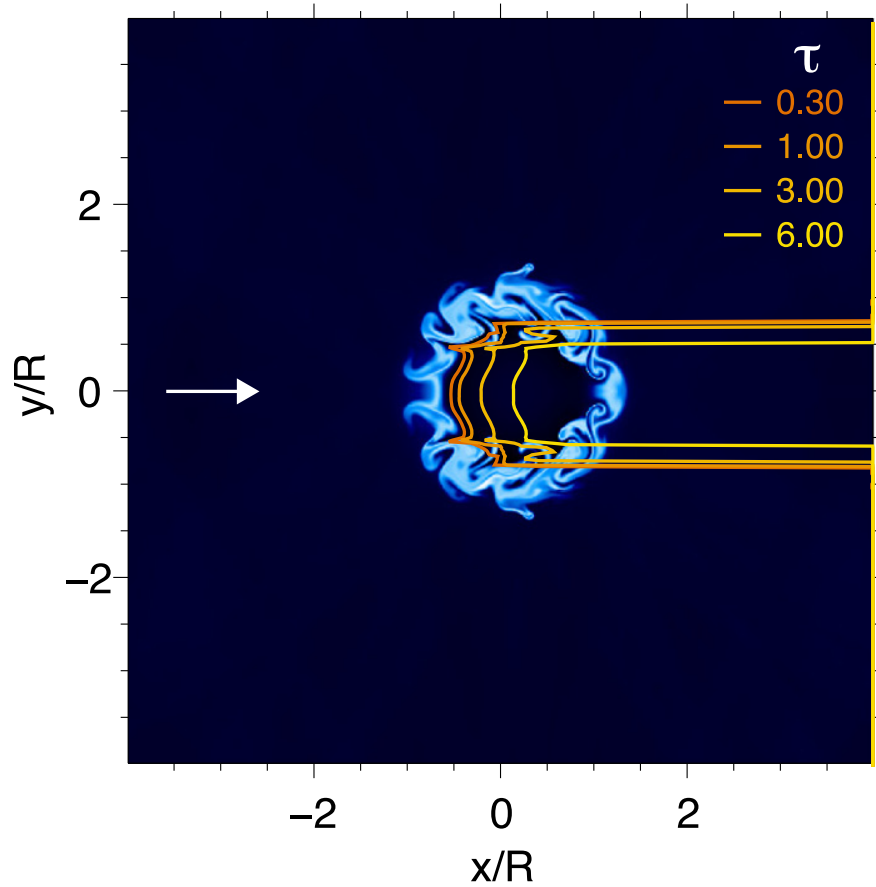

Ca II H

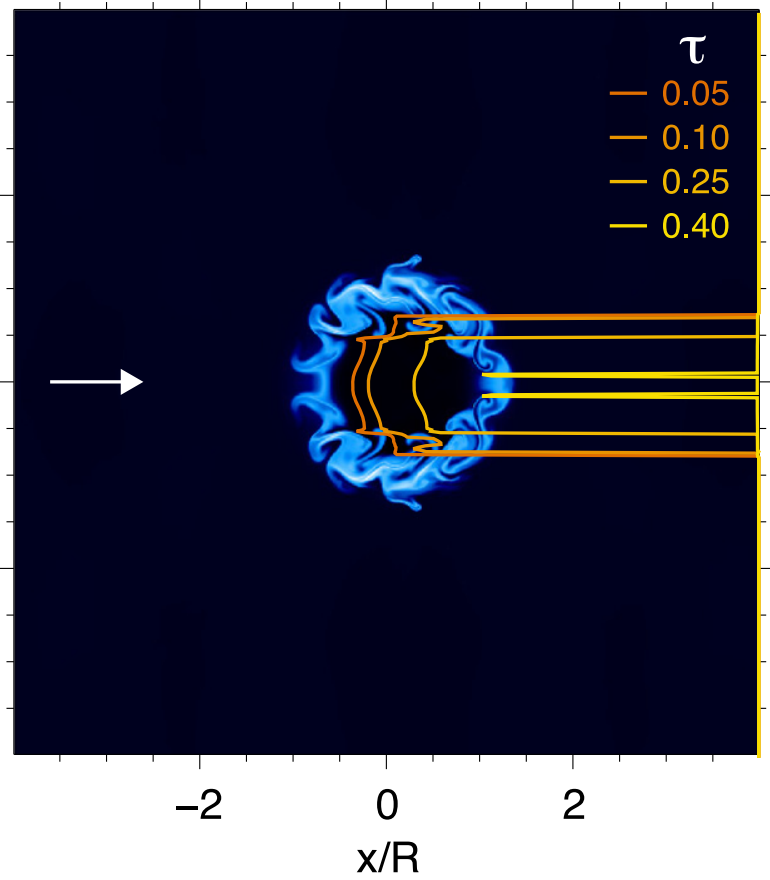

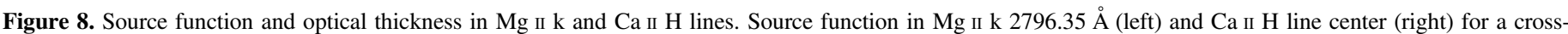

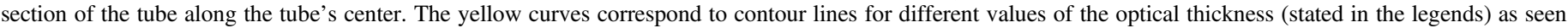
from the top (as indicated by the arrow), corresponding to an LOS angle of $0^{\circ}$. The axes are set to match those in the numerical simulation in Paper II.

ratios close to 2 because they are purely optically thin. Ratios between 1.5 and 2 are thus obtained for spatially broad rays (simulating the case of coarse instrument spatial resolution).

An important result from our forward modeling with $\mathrm{RH}$ is that the emergent intensity in both the $\mathrm{Mg}$ II and $\mathrm{Ca}$ II $\mathrm{H}$ lines comes from the boundary of such flux tubes, where resonant absorption and the KHI vortices take place. Furthermore, the optical thickness values in Figure 8 show that the boundary of such flux tubes is optically thin in both lines, while the cool and dense core is significantly opaque only in $\mathrm{Mg}$ II $\mathrm{h}$ and $\mathrm{k}$, an important difference with respect to $\mathrm{Ca}$ II $\mathrm{HK}$. This result implies that the former lines suffer less from LOS projection effects than the latter, and are therefore more favorable for prominence analysis at the limb such as that performed here.

This result allows us to perform an approximation to optically thick radiation with FoMo, as explained in Paper II, which retains the key aspects of the radiative transfer calculations. This approximation allows us to forward model swiftly and thus study in detail the observational signatures of our numerical model.

\subsection{Interpretation of the Observational Results: Resonant Absorption and KHI}

While the phase drift produced by resonant absorption that results in the transverse MHD wave model (which would correspond to the initial part of phase mixing) could potentially explain the out-of-phase difference found in our observations, it also poses a problem of detectability, and it especially fails to explain the strong dynamical coherence explained in Section 3.3. Indeed, resonant absorption is expected to be confined to the fine resonant layer, which is completely invisible to the current instrumentation. Even in inhomogeneous flux tubes with irregular internal density profiles, while the system still evolves with a main oscillating frequency, and a large-scale resonance pattern sets in around the ensemble of inhomogeneities, the complicated resonant layers are still found to be highly spatially confined (Terradas et al. 2008b; Pascoe et al. 2011; this case is sketched in Figure 7(b) and we refer to it as the multiple kink wave scenario). Such systems could potentially explain the POS dynamical coherency (assuming that the inhomogeneities within flux tubes could correspond to the observed threads) but would fail at explaining the LOS velocity coherency and especially the out-of-phase behavior.

This is where the second mechanism in our simulations, the KHI becomes important. Indeed, as explained in Section 3.4, the instability extracts energy from the resonant layer and imparts momentum on the generated eddies, which rapidly degenerate into turbulent-like flows that are still embedded in the large-scale azimuthal flow. This results in a spatial broadening of the transition layer between the prominence core and the external environment, which mostly retains the characteristic phase drift of the resonance layer with respect to the transverse displacement of the prominence core. Furthermore, the layer affected by the KHI, which occupies most of the original flux tube, is subject to significant heating, leading to a fade out of $\mathrm{Mg}$ II $\mathrm{k}$ emission, and gradual appearance of $\mathrm{Si}$ IV emission. This is shown in Figure 6, where the Doppler signal captured by the slits (placed a distance of a diameter away from the flux tube, above and below) shows the characteristic out-of-phase behavior with respect to the POS motion of the prominence core. As explained in Paper II, this is not only the result of the KHI broadening, but also of the PSF width of IRIS (which has been taken into account in our forward modeling), which spreads the signal over a significant distance. Furthermore, due to the significantly large perpendicular distance over which the LOS velocity is retained and to 
the symmetric Doppler profile with respect to the flux tube axis, the dynamical coherence in the observations is recovered.

As shown in Paper II, the characteristic observational signatures of the transverse MHD wave model can be seen for basically any LOS in a plane perpendicular to the tube axis, and for optically thin and moderately thick conditions (Paper II). In the multiple kink wave scenario previously discussed, the same observational features would be expected since the ensemble of "threads" (enhanced density regions within the large flux tube) evolve coherently, developing a large azimuthal flow around the system. The onset of the KHI around this flux tube would therefore also produce the same characteristic features as in the present case.

Another feature predicted by our numerical model is the appearance of thread-like structure in both chromospheric and transition region lines, which result from the KHI vortices and LOS projection effects. Such structure can be seen in Figure 6 and is similar to the strand-like structure obtained in the coronal case (Antolin et al. 2014). In Paper II, we discuss the possibility of the observed $\mathrm{Ca}$ II $\mathrm{H}$ threads being the KHI vortices.

\subsection{Other Interpretations}

Our detailed modeling shows that the observations are fully compatible with a state-of-the-art numerical model of transverse MHD oscillations and thus our interpretation of resonant absorption. Furthermore, we can also prove that in the scenario of other possible processes, such as rotation and the torsional Alfvén wave, the observed coherence and phase difference between the POS motion and LOS velocity cannot be reproduced.

Let us first consider the case of a torsional Alfvén wave. We can further divide this case into two different cases that would lead to the observed POS oscillation of threads. Case 1 (sketched in Figure 7(c)) corresponds to a scenario in which the ensemble of threads is subject to an $m=0$ torsional Alfvén wave (as belonging to a larger flux tube). Case 2 (sketched in Figure 7(d)) shows a single thread and corresponds to a scenario in which each thread is subject to a coupled torsional Alfvén wave and kink wave, in which the azimuthal flow from the former dominates that of the latter one. In each sketch, a generic location for the IRIS slits has been taken, in which the width of the slit is set according to the size of the thread, as is observed. Case 1 can be further divided into two cases, depending on the size of the flux tube with respect to the size of the region scanned by the rasters. If the slit positions cover different parts of the flux tube (edges and center, as pictured by case 1 within Figure 7(c)) the LOS velocity signals recovered by the green and red slit positions should be out of phase, disagreeing with the observed transverse coherence in the Doppler signals shown in Figure 5 (in each panel of this figure the signal from a slit follows the same behavior regardless of whether it is placed above or below the thread). If the slit positions cover only one half of the flux tube, the transverse coherence is recovered (as shown by case 2 in panel (c)), as well as the $180^{\circ}$ phase difference. However, in this case, a gradual increase of amplitude should be observed between the slits the closer they are to the flux tube boundary, which is not observed in our Hinode-IRIS measurements, as shown in Figure 5 in all panels. For the case of coupled torsional Alfvén and kink waves the Doppler shift will drastically change sign depending on where the slit is across the thread (as in the first case of the torsional Alfvén wave), which impedes the collective in-phase signals between the slits that we observe in Figure 5 in all panels (asymmetric $\lambda-y$ profile, cf. Section 4.3 in Paper II). This is also shown by Goossens et al. (2014), in their Figure 9.

Let us now consider the case of rotation. Similarly, we can identify two cases leading to the observed POS oscillation. The first case (sketched in Figure 7(e)) corresponds to a scenario in which the ensemble of threads is rotating coherently. In the second case (sketched in Figure 7(f)) each thread is subject to rotation coupled to a kink wave, in which the azimuthal flow of the former dominates over that of the latter one. In both cases, a slit placed always on one side of the thread should not change sign in time. Also, a slit that crosses the thread should exhibit a change in the sign of the Doppler signal. None of the observed cases in Figure 5 satisfies both of these scenarios, which invalidates rotation as well.

We note that the previous predictions should not be affected by optical thickness. Indeed, for both cases of rotation and a torsional Alfvén wave, the frontside and backside of the flux tube with respect to the observer exhibit a coherent motion. Therefore, regardless of how opaque the core of the flux tube is, the signal detected by IRIS will retain its Doppler shift value. The predictions should therefore hold, excluding both scenarios of rotation and the torsional Alfvén wave.

Figure 9 summarizes the observed features together with the expected observational features of the six considered cases. The plus/minus sign denotes a change in the LOS velocity. The green and red curves denote the shape of the LOS velocity in time, as detected by the slits of the same color in the sketches.

\section{CONCLUSIONS}

Observations of coronal transverse oscillations were first (and are often) reported in the aftermath of flares (Nakariakov et al. 1999). Such reports have been the subject of several dozen follow-up theoretical and observational studies, which suggest resonant absorption as the most plausible mechanism causing the damping of such oscillations (Nakariakov \& Verwichte 2005; De Moortel \& Nakariakov 2012). However, to date, no solid consensus exists on the causes of this damping. Furthermore, these large-scale oscillations occur only in the aftermath of an impulsive event such as a flare. Such events cannot be the main mechanism of heating in the quiescent corona because they are too infrequent (compared to the typical coronal cooling times). Heating from Alfvénic waves invoking resonant absorption in prominences or coronal loops has also previously been reported (Ofman \& Mouradian 1996; Ofman et al. 1998; Nakariakov et al. 1999), supported by numerous theoretical studies suggesting that this mechanism is efficient and ubiquitous (Arregui et al. 2008; Terradas et al. 2008b; Goossens et al. 2011). However, such observational reports have been largely based on indirect evidence due to incomplete measurements (lack of spectroscopic information allowing for the detection of the three-dimensional flow and heating generated by the wave) or a lack of resolution leading to a precarious causal relation between resonant absorption and the subsequent heating. In the present work, we show for the first time direct evidence of resonant absorption as measured with a spectrograph and associated heating to transition region temperatures. This association is performed with unprecedented detail and therefore narrows down to unparalleled levels the causal relation in evidence for wave heating. 


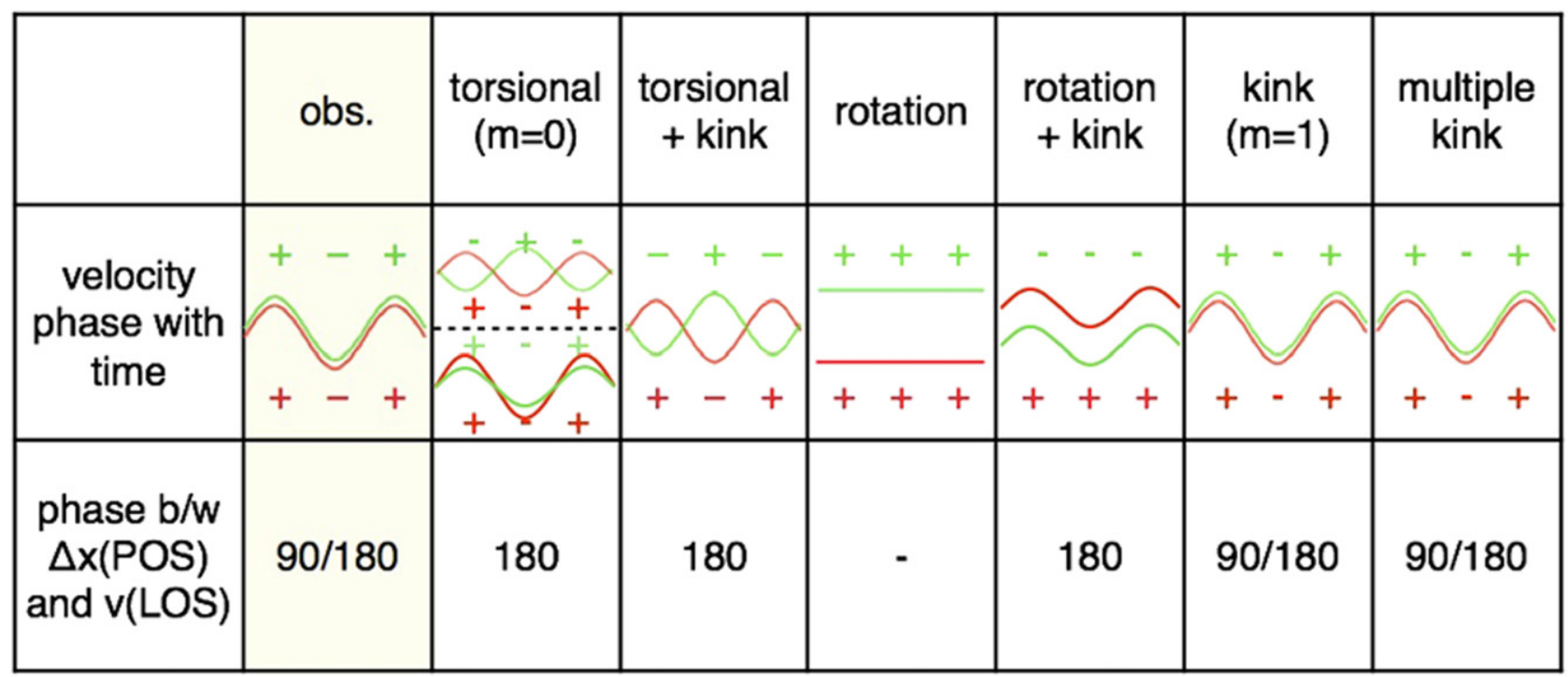

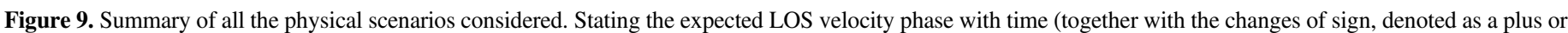

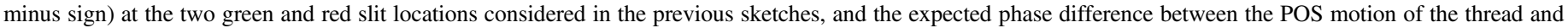

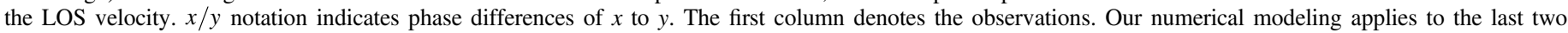
columns. These are the only cases among those considered above that correctly match the observed features.

Our numerical modeling suggests that an important coupling between resonant absorption and KHI takes place, in which KHI efficiently extracts energy from the resonant layer and converts it into heat. As the KHI sets in, viscous and ohmic heating strongly increase due to the generation of vortices and current sheets around most of the flux tube (Goossens et al. 2002). As explained previously, the heating occurs rapidly at first and proceeds to a roughly constant rate for most of the simulation. The boundary layer, characterized by transition region temperatures around $10^{5} \mathrm{~K}$, expands rapidly in the timescale of one period, mostly inward toward the center of the tube, which leads to fading with time of threads in $\mathrm{Ca}$ II and Mg II (both 10,000 K), and the subsequent appearance (in the same location) of threads at higher temperatures on a timescale of 10-15 minutes (e.g., Si IV at 80,000 K) (Figure 6). Such heating is also present in our observations on similar timescales: as the moving threads in the $\mathrm{Ca}$ II line fade away (see the animation of Figure 1), co-spatial threads appear in the hotter Si IV line with similar horizontal speeds. Space-time plots of the Ca II and Si IV images show this clearly (Figure 3). Even though some locations show coexistence of cooler and hotter materials, the trajectories of several threads show a transition from lower to higher temperatures with threads finally fading out even from Si IV images. This scenario cannot be explained by a multithermal structure of the threads, and is strongly suggestive of cool plasma being heated to coronal temperatures through the mid-temperature range of the Si IV line. Evidence for coronal counterparts of the prominence comes from the animation of Figure 1: while clear fine structures cannot be identified in the Fe IX $(\sim 1 \mathrm{MK})$ channel of $S D O /$ AIA, horizontally elongated bright features do occupy the same region as in the cooler passbands (Figure 1).

The observed heating is directly predicted by our transverse MHD wave model and thus provides supporting evidence for our interpretation, which, as mentioned, is focused on the spatial coherence and the phase relationships between LOS velocity and POS oscillations. The combination of observations and modeling thus provide compelling evidence for wave dissipation through the combination of resonant absorption and $\mathrm{KHI}$ and the result of heating to at least transition region temperatures in a prominence. The observed oscillations have an energy flux of $100-1000 \mathrm{~W} \mathrm{~m}^{-2}$, where the velocity amplitude is $20 \mathrm{~km} \mathrm{~s}^{-1}$ in the case in which the view angle is $45^{\circ}$, the plasma density is typically $10^{-11}-10^{-10} \mathrm{~kg} \mathrm{~m}^{-3}$ (Labrosse et al. 2010), and the Alfvén speed is assumed to be $300-600 \mathrm{~km} \mathrm{~s}^{-1}$. Here we have considered the more general case of the multiple kink wave scenario (sketched in Figure 7(b)), for which the energy flux is within a factor of two from that of the bulk Alfvén wave multiplied by a filling factor (Goossens et al. 2013; van Doorsselaere et al. 2014). We take a conservative filling factor of $5 \%$ to avoid overestimation. The energy flux is enough to locally heat the plasma to coronal temperatures (Withbroe \& Noyes 1977). Our results may also provide insight into the puzzling rapid disappearance of prominences (Tandberg-Hanssen 1995) and in the heating mechanism of hot plasma bubbles that have been reported in prominences (Berger et al. 2011). More generally, our results provide a pathfinder to search for heating resulting from resonant absorption in other solar regions (e.g., quiet corona and coronal holes) that are permeated with transverse magnetic waves.

Hinode is a Japanese mission developed and launched by ISAS/JAXA, with NAOJ as a domestic partner and NASA and STFC (UK) as international partners. It is operated by these agencies in cooperation with ESA and NSC (Norway). IRIS is a NASA small explorer mission developed and operated by LMSAL with mission operations executed at NASA Ames Research center and major contributions to downlink communications funded by NSC through an ESA PRODEX contract. $S D O$ is part of NASA's Living With a Star Program. Numerical computations were carried out on Cray XC30 at the Center for Computational Astrophysics, NAOJ. T.J.O. thanks Ipponkakou-kai for their encouragement and was supported by JSPS KAKENHI grant No. 25800120 (PI: T.J.O.). T.J.O. and P.A. were supported by JSPS KAKENHI grant No. 25220703 (PI: S. Tsuneta). B.D.P. was supported by NASA under contracts 
NNG09FA40C (IRIS), NNX11AN98G, and NNM12AB40P. T.V.D. was supported by FWO Vlaanderen's Odysseus programme, GOA-2015-014 (KU Leuven) and the IAP P7/ 08 CHARM (Belspo).

\section{REFERENCES}

Alfvén, H. 1947, MNRAS, 107, 211

Andries, J., Arregui, I., \& Goossens, M. 2005, ApJL, 624, L57

Anfinogentov, S., Nisticò, G., \& Nakariakov, V. M. 2013, A\&A, 560, AA107

Antolin, P., Okamoto, T. J., De Pontieu, B., et al. 2015, ApJ, 809, 72 (Paper II)

Antolin, P., \& Shibata, K. 2010, ApJ, 712, 494

Antolin, P., \& van Doorsselaere, T. 2013, A\&A, 555, AA74

Antolin, P., \& Verwichte, E. 2011, ApJ, 736, 121

Antolin, P., Yokoyama, T., \& van Doorsselaere, T. 2014, ApJL, 787, L22

Arregui, I., Oliver, R., \& Ballester, J. L. 2012, LRSP, 9, 2

Arregui, I., Soler, R., Ballester, J. L., \& Wright, A. N. 2011, A\&A, 533, AA60

Arregui, I., Terradas, J., Oliver, R., \& Ballester, J. L. 2007, A\&A, 466, 1145

Arregui, I., Terradas, J., Oliver, R., \& Ballester, J. L. 2008, ApJL, 682, L141

Aschwanden, M. J., Fletcher, L., Schrijver, C. J., \& Alexander, D. 1999, ApJ, 520,880

Aschwanden, M. J., Nightingale, R. W., Andries, J., Goossens, M., \& van Doorsselaere, T. 2003, ApJ, 598, 1375

Auer, L. 2003, Stellar Atmosphere Modeling, 288, 3

Berger, T., Testa, P., Hillier, A., et al. 2011, Natur, 472, 197

De Moortel, I., \& Nakariakov, V. M. 2012, RSPTA, 370, 3193

De Pontieu, B., McIntosh, S. W., Carlsson, M., et al. 2007, Sci, 318, 1574

De Pontieu, B., Title, A. M., Lemen, J. R., et al. 2014, SoPh, 289, 2733

Dere, K. P., Landi, E., Mason, H. E., Monsignori Fossi, B. C., \& Young, P. R. 1997, A\&AS, 125, 149

Dere, K. P., Landi, E., Young, P. R., et al. 2009, A\&A, 498, 915

Goossens, M., Andries, J., \& Aschwanden, M. J. 2002, A\&A, 394, L39

Goossens, M., Erdélyi, R., \& Ruderman, M. S. 2011, SSRv, 158, 289

Goossens, M., Hollweg, J. V., \& Sakurai, T. 1992, SoPh, 138, 233

Goossens, M., Soler, R., Terradas, J., van Doorsselaere, T., \& Verth, G. 2014, ApJ, 788, 9

Goossens, M., van Doorsselaere, T., Soler, R., \& Verth, G. 2013, ApJ, 768, 191

Grevesse, N., \& Anders, E. 1991, in Solar Interior and Atmosphere, ed. A. N. Cox, W. C. Livingston, \& M. S. Matthews (Tucson, AZ: Univ. of Arizona Press), 1227

Handy, B. N., Acton, L. W., Kankelborg, C. C., et al. 1999, SoPh, 187, 229

He, J.-S., Tu, C.-Y., Marsch, E., et al. 2009, A\&A, 497, 525

Hillier, A., Morton, R. J., \& Erdélyi, R. 2013, ApJL, 779, LL16

Hollweg, J. V., Yang, G., Cadez, V. M., \& Gakovic, B. 1990, ApJ, 349, 335

Ionson, J. A. 1978, ApJ, 226, 650

Karpen, J. T., Antiochos, S. K., Dahlburg, R. B., \& Spicer, D. S. 1993, ApJ, 403, 769

Kosugi, T., Matsuzaki, K., Sakao, T., et al. 2007, SoPh, 243, 3

Kunasz, P., \& Auer, L. H. 1988, JQSRT, 39, 67

Labrosse, N., Heinzel, P., Vial, J.-C., et al. 2010, SSRv, 151, 243

Lapenta, G., \& Knoll, D. A. 2003, SoPh, 214, 107

Leenaarts, J., Pereira, T. M. D., Carlsson, M., Uitenbroek, H., \& De Pontieu, B. 2013, ApJ, 772, 90
Lemen, J. R., Title, A. M., Akin, D. J., et al. 2012, SoPh, 275, 17

Lin, Y. 2011, SSRv, 158, 237

Lin, Y., Soler, R., Engvold, O., et al. 2009, ApJ, 704, 870

Mackay, D. H., Karpen, J. T., Ballester, J. L., Schmieder, B., \& Aulanier, G. 2010, SSRv, 151, 333

Mathioudakis, M., Jess, D. B., \& Erdélyi, R. 2013, SSRv, 175, 1

Matsumoto, T., \& Suzuki, T. K. 2014, MNRAS, 440, 971

McIntosh, S. W., De Pontieu, B., Carlsson, M., et al. 2011, Natur, 475, 477

Nakariakov, V. M., \& Ofman, L. 2001, A\&A, 372, L53

Nakariakov, V. M., Ofman, L., Deluca, E. E., Roberts, B., \& Davila, J. M. 1999, Sci, 285, 862

Nakariakov, V. M., \& Verwichte, E. 2005, LRSP, 2, 3

Nisticò, G., Nakariakov, V. M., \& Verwichte, E. 2013, A\&A, 552, AA57

Ofman, L., Davila, J. M., \& Steinolfson, R. S. 1994, GeoRL, 21, 2259

Ofman, L., Kucera, T. A., Mouradian, Z., \& Poland, A. I. 1998, SoPh, 183, 97

Ofman, L., \& Mouradian, Z. 1996, A\&A, 308, 631

Ofman, L., \& Wang, T. J. 2008, A\&A, 482, L9

Okamoto, T. J., \& De Pontieu, B. 2011, ApJL, 736, L24

Okamoto, T. J., Tsuneta, S., Berger, T. E., et al. 2007, Sci, 318, 1577

Oliver, R. 2009, SSRv, 149, 175

Parnell, C. E., \& De Moortel, I. 2012, RSPTA, 370, 3217

Pascoe, D. J., Wright, A. N., \& De Moortel, I. 2010, ApJ, 711, 990

Pascoe, D. J., Wright, A. N., \& De Moortel, I. 2011, ApJ, 731, 73

Pesnell, W. D., Thompson, B. J., \& Chamberlin, P. C. 2012, SoPh, 275, 3

Rybicki, G. B., \& Hummer, D. G. 1992, A\&A, 262, 209

Sakurai, T., Goossens, M., \& Hollweg, J. V. 1991, SoPh, 133, 227

Schmieder, B., Kucera, T. A., Knizhnik, K., et al. 2013, ApJ, 777, 108

Schrijver, C. J., Title, A. M., Berger, T. E., et al. 1999, SoPh, 187, 261

Shimizu, T., Nagata, S., Tsuneta, S., et al. 2008, SoPh, 249, 221

Soler, R., Ruderman, M. S., \& Goossens, M. 2012, A\&A, 546, AA82

Soler, R., Terradas, J., Oliver, R., Ballester, J. L., \& Goossens, M. 2010, ApJ, 712,875

Suematsu, Y., Tsuneta, S., Ichimoto, K., et al. 2008, SoPh, 249, 197

Tandberg-Hanssen, E. 1995, The Nature of Solar Prominences (Dordrecht: Kluwer)

Terradas, J., Andries, J., Goossens, M., et al. 2008a, ApJL, 687, L115

Terradas, J., Arregui, I., Oliver, R., et al. 2008b, ApJ, 679, 1611

Tomczyk, S., \& McIntosh, S. W. 2009, ApJ, 697, 1384

Tomczyk, S., McIntosh, S. W., Keil, S. L., et al. 2007, Sci, 317, 1192

Tsuneta, S., Ichimoto, K., Katsukawa, Y., et al. 2008, SoPh, 249, 167

Uchida, Y., \& Kaburaki, O. 1974, SoPh, 35, 451

Uitenbroek, H. 1989, A\&A, 213, 360

Uitenbroek, H. 1997, SoPh, 172, 109

Uitenbroek, H. 2001, ApJ, 557, 389

van Ballegooijen, A. A., Asgari-Targhi, M., Cranmer, S. R., \& DeLuca, E. E. 2011, ApJ, 736, 3

van Doorsselaere, T., Andries, J., Poedts, S., \& Goossens, M. 2004, ApJ, 606,1223

van Doorsselaere, T., Gijsen, S. E., Andries, J., \& Verth, G. 2014, ApJ, 795, 18

Vernazza, J. E., Avrett, E. H., \& Loeser, R. 1981, ApJS, 45, 635

Verth, G., Terradas, J., \& Goossens, M. 2010, ApJL, 718, L102

Vial, J.-C., \& Engvold, O. 2015, Solar Prominences (Astrophysics and Space Science Library, Vol. 415; Dordrecht: Springer)

Wang, T., Ofman, L., Davila, J. M., \& Su, Y. 2012, ApJL, 751, LL27

Withbroe, G. L., \& Noyes, R. W. 1977, ARA\&A, 15, 363 